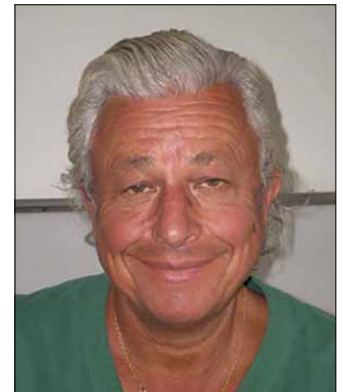

Marc BERT

Rédacteur en chef

\section{La formation continue}

a formation continue est une nécessité professionnelle, nécessité encore accrue dans les métiers de la santé à l'évolution rapide, tant sur le plan des connaissances que sur celui des applications cliniques. Cette nécessité est même devenue une obligation, malheureusement encore mal contrôlée, certains praticiens pouvant exercer de nombreuses années sans suivre le moindre cours, la moindre conférence.

Cette formation continue est assurée par de nombreux organismes, certains liés à l'université, d'autres privés et son financement peut être pris en charge par l'Etat, essentiellement au travers des impôts que les contribuables que nous sommes sont dans l'obligation de payer.

Il est donc normal qu'un contrôle de ces formations soit effectué !

Titulaire depuis 30 ans d'une licence de pilote privé d'avion, j’ai le plaisir d'avoir accès à un univers fascinant, mais évoluant rapidement, tant au niveau des machines qu'à celui de la réglementation. Cette licence n'est donc valable que 2 ans et son renouvellement passe par le contrôle des connaissances du pilote, permettant essentiellement de vérifier sa capacité à gérer des situations difficiles. Ce renouvellement s'effectue au travers d'un vol au cours duquel un instructeur dûment qualifié s'assure, de visu, que le pilote est apte à continuer de voler et d'emmener des passagers.

Il semblerait logique que le contrôle des formations continues professionnelles s'inscrive dans une démarche similaire : vérifier de visu que le cours correspond à un besoin, que les participants à ce cours soient satisfaits et qu'il leur apporte « un plus » dans leur activité professionnelle. Grave erreur!

Ayant le plaisir de donner depuis plusieurs années une formation sur l'occlusion en général et en implantologie en particulier, dans le cadre d'un organisme de formation continue, j'ai constaté, en lisant les questionnaires de fin de cours, qu'il donnait satisfaction à plus de $95 \%$ des participants. Cette formation a été soumise à l'Agence Nationale du Développement Professionnel Continu (ANDPC), en charge du financement par l'Etat de ces formations. Et là, surprise ! Un obscur Comité Scientifique, dont certains membres ont un curriculum vitae désespérément vide, a refusé le financement de cette formation, se basant sur des éléments comme quelques diapositives de ce cours, sans leur commentaire ni les animations qui les rendent claires et un résumé de quelques pages d'un cours faisant 6 heures. Un membre du Comité a-t-il suivi le cours ? Bien sur que non! A-t-on tenu compté de l'avis des praticiens ayant suivi la formation? Que nenni, ces galeux, ces pelés!

Il existe donc 2 catégories de praticiens : ceux n'ayant pas suivi la formation et l'ayant trouvée mauvaise, et ceux l'ayant suivie et l'ayant trouvée de qualité. Eh bien, au pays de Descartes, de Voltaire et de son Éloge de la Raison », ce sont les premiers qui sont décisionnaires...

Ainsi va la formation continue dans notre beau pays... 